\title{
Análisis histológico de lesiones pódales de pollos comerciales en la Planta de Sacrificio de Pollo Olympico S.A., Colombia
}

\author{
Histological analyses of toe lesions for commercial broiler at the \\ slaughterhouse of Pollo Olympico S.A., Colombia.
}

Ricardo Balaguera ${ }^{1}$, Geovanna M. Córdoba²

\section{Resumen}

Objetivo: Realizar el análisis histológico de lesiones pódales de pollos comerciales en la planta de sacrificio de Pollo Olympico S.A., Colombia con el fin de discutir aspectos sobre su retención sanitaria en plantas de sacrificio. Materiales y métodos: Se utilizó la población avícola de 10 granjas de la empresa Pollo Olympico S.A evaluando macroscópicamente 129.551 animales y microscópicamente 330 lesiones podales. El análisis histológico evaluó el tipo y la profundidad de las lesiones inflamatorias y la presencia de estructuras micóticas. Resultados: La evaluación macroscópica evidenció lesiones podales en el 64,7\% de la población total de aves; la microscópica mostró un predominio de procesos inflamatorios con presentación de heterófilos (73,1\%), el 19\% fueron infiltrados mixtos y el 7.9\% de predominio mononuclear. Las lesiones encontradas mostraron una localización principalmente subcorneal (77.9\%) y la ausencia de estructuras micóticas, células gigantes o lesiones vasculares.

Palabras clave: pododermatitis aviar, inocuidad alimentaria, retención de piezas en plantas de sacrificio, morfología inflamatoria de lesiones podales.

\section{Abstract}

Objetive: To conduct histological analyses of toe lesions for commercial broiler at the slaughterhouse of Pollo Olympico S.A., Colombia, with the ultimate goal of proposing recommendations about sanitary containment. Materials and Methods: Poultry subjects were sampled in 10 farms of Pollo Olympico S.A. Macroscopic inspections were conducted on 129.551 animals while the microscopic ones were on 330 toe lesions. Histological analyses evaluated type and depth of inflammatory lesions as well as the presence of fungal infections Results: Macroscopic analyses revealed inflammatory process mediated by heterophiles $(73,1 \%)$, mixed infiltrates (19\%) and mononuclear (7.9\%). Lesions were mainly localized at the sub-corneal level (77.9\%) and showed no signs of fungal structures, giant cells or vascular presence.

Keywords: bird toe-pad-dermatitis, food safety assessment, containment at slaughterhouses, morphology of inflamatory lesions.

Docente -investigador Universidad Santo Tomas y Fundación Universitaria San Martin. ribal05@gmail.com 


\section{Introducción}

La pododermatitis plantar es un cuadro multi sistémico que puede ocasionar cojeras y pérdida del equilibrio en aves, con consecuencias tanto económicas como de bienestar animal. Se han realizado estudios para determinar los diferentes factores que intervienen en la presentación de problemas podales, reportando; causas de origen viral, genéticas, y micóticas. Las causas más frecuentemente reportadas son factores bacterianos, nutricionales, estrés calórico y especialmente, las relacionadas con las condiciones de la cama de los animales (1-10).

La pododermatitis es una lesión del cojinete plantar común en pollos de engorde criados sobre camas húmedas y apelmazadas, los materiales de las camas y la humedad de ésta juegan un papel importante, $(11,12)$.

El reaprovechamiento de la cama hasta por cuatro veces consecutivas, práctica popularizada en una gran parte de los productores de pollos de engorde, favorece la humedad y la compactación, lo que contribuye a una mayor ocurrencia de lesiones podales de forma independiente a la densidad de población en los galpones (13). Pizarro et al (14) reportan que las camas tratadas con aluminosilicato pueden neutralizar el amoniaco liberado durante las primeras cuatro semanas de crianza.

Uno de los indicadores del bienestar animal (BA) en aves es la ausencia de lesiones en la canal principalmente pododermatitis, debido a que este problema se relaciona particularmente con las condiciones de alojamiento y manejo $(15,16)$. Los problemas de BA se están incrementando de forma importante, incluso llegando a ser un cuello de botella en la legislación de algunos países (7).

Por otra parte, se han definido diferentes grados de lesión plantar. Para este estudio se utilizó la clasificación de Remple, et al (17) y Halliwell et al (18):
Tipo $O$. No presenta lesión.

Tipo I. Es el inicio de una lesión en el cojinete plantar. Normalmente está localizada a nivel superficial, es de característica inflamatoria leve, existe integridad en el epitelio de cubierta.

Tipo II. Es un estado avanzado crónico. Aparece reacción fibrosa y aumenta el proceso inflamatorio, puede empezar a aparecer signos de infección.

Tipo III. Se distinguen deformaciones y una reacción fibrosa abundante. Es muy común la aparición de osteomielitis con compromiso articular grave. La lesión puede ser proliferativa o degenerativa con tendencia a ulceración, aparecen abscesos supurantes y marcada reacción fibrosa de los tejidos.

Aunque en algunos estudios la pododermatitis no es de las causas más frecuentes de retención de carcasas (10), otros informan correlación positiva entre el control clínico de pododermatitis y la disminución en la tasa de retenciones en plantas de sacrificio (19). En Colombia, en las plantas de proceso de aves, las pododermatitis causan pérdidas económicas debido a retenciones de estas piezas por las autoridades sanitarias, lo cual repercute a lo largo de la cadena de producción.

La alta densidad de aves en los galpones es un factor relacionado con daños en las extremidades y retenciones en las plantas de sacrificio. Sin embargo puede haber otros factores involucrados, al respecto, estudios en Europa señalan la viabilidad de utilizar marcadores de riesgo para anticipar las razones de decomiso en la inspección de la carne y con ello poder monitorear y mejorar las prácticas en diversas etapas de la producción de pollos (20-21).

Las razones de decomiso en nuestro país carecen de estudios detallados que relacionen los grados de lesión con las retenciones, siendo el único parámetro inicial de evaluación la observación visual y la sospecha sin pruebas confirmatorias de contaminación fúngica. 
A nivel microscópico las pododermatitis normalmente se inician como un proceso congestivo y edematoso produciéndose inflamación de patas y dedos, si la lesión avanza puede llegar hasta la necrosis o muerte celular (22). En general se encuentra una estrecha relación entre la severidad de la lesión macroscópica y microscópica, la morfología de los elementos micóticos y su relación con las lesiones tisulares en ocasiones presentan un patrón típico para algunas especies (23). Las lesiones micóticas se acompañan generalmente de reacciones severas granulomatosas con necrosis central.

\section{Materiales y métodos Población y muestra}

El trabajo de investigación se llevó a cabo con la participación de la Empresa Pollo Olympico.

En su desarrollo participaron 10 granjas que conforman la Empresa y están ubicadas en el municipio de Villeta (Cundinamarca). Estas granjas tienen una población total de 873.866 aves, distribuidas como se presenta la Tabla 1.

Tabla 1. Población total de aves por granja.

\begin{tabular}{|lc|}
\hline $\begin{array}{c}\text { Nombre de } \\
\text { la granja }\end{array}$ & $\begin{array}{c}\text { Población } \\
\text { de aves }\end{array}$ \\
\hline Buenavista & 130.348 \\
\hline Primavera & 69.360 \\
\hline Laureles & 86.200 \\
\hline Fredonia & 105.060 \\
\hline Ponderosa & 175.440 \\
\hline Luisa & 36.720 \\
\hline Altamira & 102.000 \\
\hline San José & 40.784 \\
\hline Troya & 56.071 \\
\hline Refugio & 71.383 \\
\hline
\end{tabular}

La población tomada para el estudio macroscópico fueron aves de lotes de las diferentes granjas, que cumplieron su ciclo productivo y llegaron a la planta de sacrificio, ubicada en Bogotá, durante 12 semanas consecutivas, esto permitía tener al menos un lote de cada granja.

Una vez sacrificadas las aves, el personal profesional de la planta evaluó y clasificó las lesiones podales a nivel macroscópico. La clasificación se realizó como se describió previamente y teniendo en cuenta muestras de todas las granjas de la empresa $(17,18)$.

Para el estudio histológico se tuvieron en cuenta las lesiones podales de la evaluación macroscópica descritas anteriormente, seleccionando en cada semana de estudio, las primeras 30 lesiones clasificadas con grados II y III. Después del procesamiento histotécnico se eliminaron el 7\% de las muestras enviadas desde la planta de sacrificio, por no tener la calidad o las características mínimas para ser evaluadas. Por lo tanto se valoraron las características inflamatorias de 330 muestras.

Se fraccionaron porciones menores de un centímetro cúbico para fijarlas en formol buferado al 10\% y procesarlas con el método de imbibición en parafina y coloración de hematoxilina- eosina. La lectura de los tejidos procesados se realizó en microscopio de luz en 10X y 40X en la Universidad Santo Tomas. Para este estudio se hizo una lectura del material obtenido y se determinó analizar los siguientes aspectos:

1. Clasificación y graduación de la morfología de las lesiones inflamatorias: presencia de inflamación de infiltrado de heterófilos, de células mononucleares o presencia de Granulomas con células epiteliodes.

2. Profundidad de la lesión; describiendo si se limita a la unión de la dermis y la epidermis o si es leve o moderadamente más profunda.

3. Identificación de estructuras micóticas con ayuda de la coloración de acido periódico de Schiff (PAS) y evaluando en los infiltrados sospechosos (reacciones granulomatosas o infiltrados mononucleares de grado severo). 


\section{Resultados}

Lesiones pódales macroscópicas. En la Tabla 2 se presentan las lesiones pódales macroscópicas observadas en las aves de estudio.

Tabla 2. Lesiones pódales macroscópicas observadas en aves de 10 granjas de Pollo Olympico S.A., Colombia.

\begin{tabular}{lccc} 
Granja & $\begin{array}{c}\text { Población } \\
\text { Observada }\end{array}$ & $\begin{array}{c}\text { Población con lesiones pódales } \\
\text { macroscópicas }\end{array}$ & Porcentaje \\
\hline Luisa & 5130 & 4105 & $80,0 \%$ \\
\hline Primavera & 4976 & 4144 & $83,3 \%$ \\
\hline San José & 2819 & 2230 & $79,1 \%$ \\
\hline Refugio & 4682 & 3741 & $79,9 \%$ \\
\hline Altamira & 14232 & 11510 & $80,9 \%$ \\
\hline Buenavista & 13097 & 10503 & $80,2 \%$ \\
\hline Troya & 22594 & 15925 & $70,5 \%$ \\
\hline Fredonia & 19543 & 11230 & $57,5 \%$ \\
\hline Laureles & 20686 & 9679 & $46,8 \%$ \\
\hline Ponderosa & 21792 & 10729 & $49,2 \%$ \\
\hline
\end{tabular}

En la Figura 1 se observan las lesiones macroscópicas pódales tipo III observadas, con múltiples focos de necrosis demarcados con piel amarillenta y reacción inflamatoria.

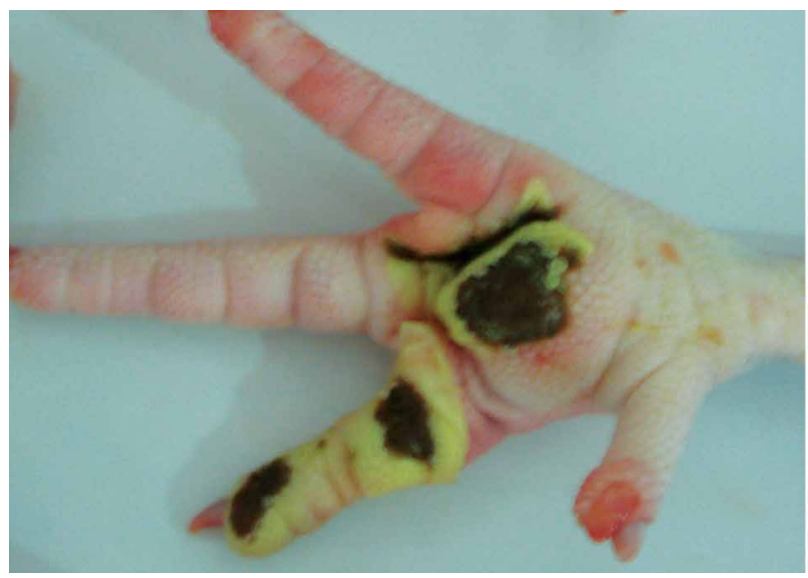

Figura 1. Lesiones macroscópicas podales tipo III.

\section{Lesiones pódales microscópicas}

En la Figura 2 se observa necrosis superficial, reacción inflamatoria subcorneal e inflamación mononuclear perivascular severa en la dermis profunda.

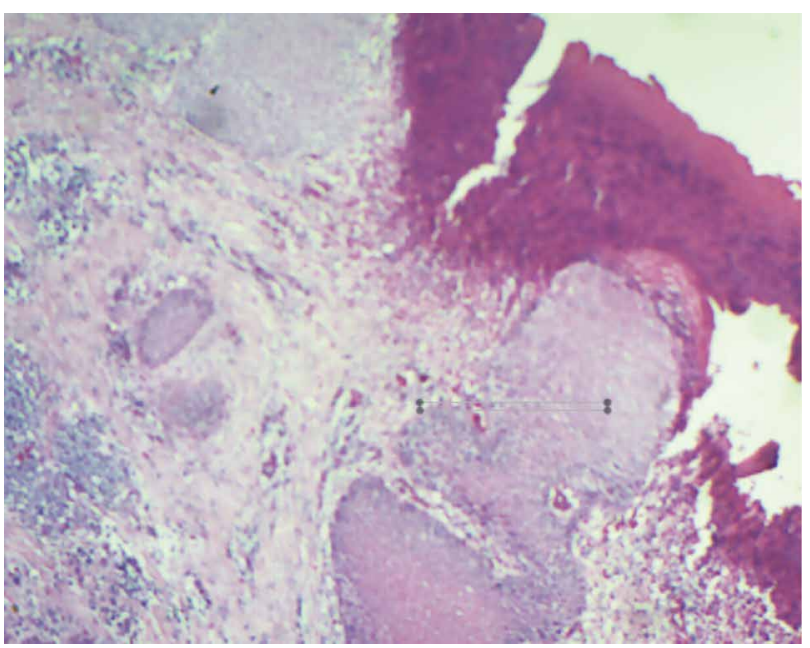

Figura 2. Lesiones microscópicas de pododermatitis. 
El infiltrado con predominio de heterófilos resultó ser el más frecuente con 241 casos, seguido de infiltrados con presencia de heterófilos y mononucleares en cantidades similares (mixtos) con $63 \mathrm{ca}-$ sos y 26 casos presentaban predominio de células mononucleares, Tabla 3 y figura 3.

Tabla 3. Número de casos y porcentajes de los tipos de inflamación de las pododermatitis observadas.

\begin{tabular}{lcc}
\multicolumn{1}{c}{ Morfología } & $\begin{array}{r}\text { Número } \\
\text { de casos }\end{array}$ & Porcentaje \\
\hline Predominio de heterófilos & 241 & $73 \%$ \\
\hline Inflamación mixta & 63 & $19 \%$ \\
\hline $\begin{array}{l}\text { Predominio de } \\
\text { mononucleares }\end{array}$ & 26 & $8 \%$ \\
\hline $\begin{array}{l}\text { Granulomas típicos } \\
\text { Total }\end{array}$ & 0 & 0 \\
\hline
\end{tabular}

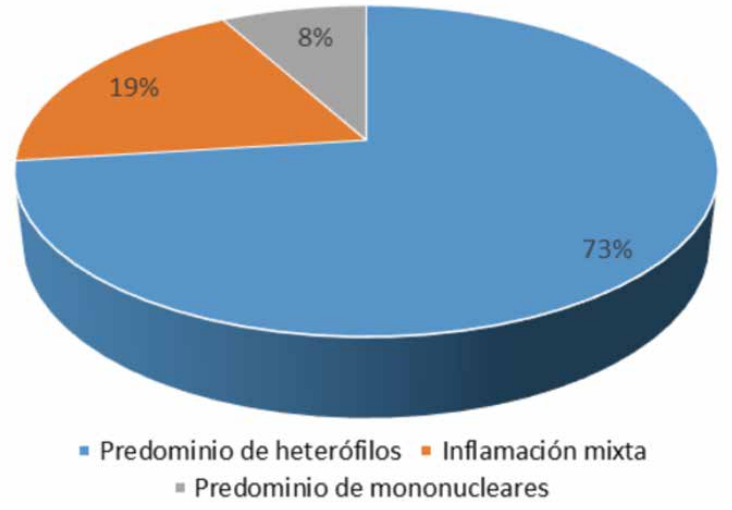

Figura 3. Número de casos en porcentajes de presentación de los tipos de inflamación de las pododermatitis observadas.

Presencia de inflamación granulomatosa crónica y de estructuras micóticas en la dermis superficial y profunda. Negativa en todos los cortes estudiados con coloración de hematoxilina y eosina. Se seleccionaron 15 casos con reacción mononuclear de moderadas a severas para utilizar con ellos la coloración de acido periódico de Schiff y evaluar detalladamente la presencia de estructuras micóticas en la dermis superficial y profunda. En estos casos el resultado también fue negativo.

\section{Profundidad de la lesión}

En cuanto a la profundidad de la lesión, el 78\% de los casos presentaron lesiones subcorneales (unión dermis epidermis), el $9 \%$ lesiones con profundidad moderada y el 13\% condiciones de dermatitis profunda, Figura 4.

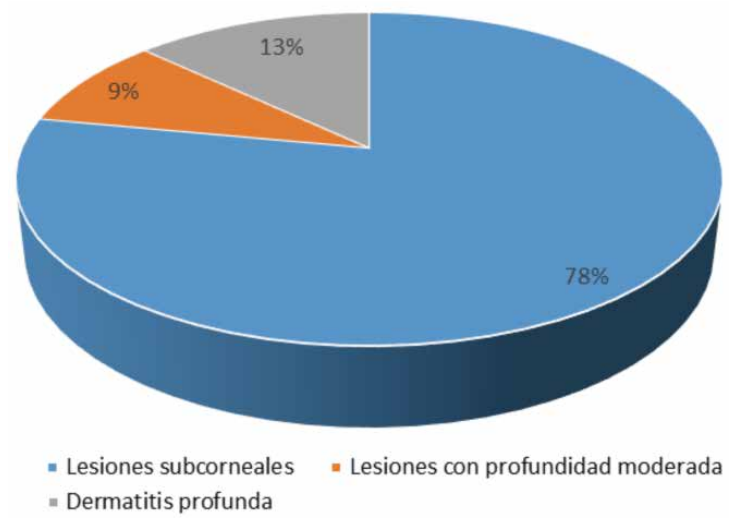

Figura 4. Porcentaje de presentación de cada uno los grados de profundidad de los procesos inflamatorios de las pododermatitis observadas.

\section{Discusión}

En el análisis histológico a nivel macroscópico se evidenció una alta presencia de problemas pódales llegando a un promedio de $64,7 \%$ de la población avícola total de la empresa y alcanzando el $83,3 \%$ en la granja primavera como ejemplo de la mayor presentación individual por granja. Al respecto, De Queiroz, et al (22) reportó en Brasil resultados similares con porcentajes entre $20 \%$ a $80 \%$; Ekstrand y col (20) reportaron en un estudio en Suecia un 32\% de lesiones moderadas más un 6\% de lesiones severas. Así mismo, Pagazaurtundua y et al (21) en el Reino Unido describen un lote con $92 \%$ de presentación de pododermatitis, pero con un $16 \%$ de presentación promedio en 178 lotes del mismo estudio. Oviedo y et al (24) reportan una prevalencia $1 \%$ a 3\% anotando que los problemas podales son las causas más frecuentes de eliminación y aumentan la mortalidad tardía en aves. 
En el análisis histológico a nivel microscópico se observó degeneración hidrópica de células epiteliales, infiltración heterofilia subcórneal, hiperemia e infiltración mononuclear en la dermis adyacente, acantosis e hiperqueratosis, estos resultados son comparables con los reportados por Queiroz y Santos (22-9). Así mismo, el infiltrado con predominio de heterófilos resulto ser el más frecuente, seguido de infiltrados con presencia de heterófilos y mononucleares en cantidades similares (mixtos) y por último el predominio de células mononucleares.

En los hallazgos microscópicos de pododermatitis se encuentra una estrecha relación con lo observado macroscópicamente, las lesiones necróticas y el infiltrado superficial de heterófilos; la acantosis y la hiperqueratosis están presente en el $100 \%$ de las muestras analizadas.

Es importante resaltar que en todos los cortes estudiados con coloración de hematoxilina y eosina, así como con la coloración de ácido periódico de Schiff no se evidencia presencia de inflamación granulomatosa crónica y de estructuras micóticas en la dermis superficial y profunda, estos resultados son similares a los reportados por Mejia (25) y Olias (26) quienes reportan escasa presentación de estos agentes patógenos en los tejidos internos, incluso afirman que la Aspergilosis, relativamente frecuente, difícilmente se presenta en órganos diferentes al pulmón.

Con el análisis de la severidad de las lesiones, la búsqueda de hongos dentro de los tejidos internos y la profundidad de las lesiones se quiso dar continuidad a un estudio anterior que analizó varios factores de calidad de estas estructuras susceptibles de retención en la planta de sacrificio y en el que Gómez et al (27), recomiendan realizar estudios para demostrar la integridad y funcionalidad del tejido, con el fin de establecer cómo esos órganos se encuentran afectados y evitar pérdidas por producto decomisado.
Teniendo en cuenta que los tejidos evaluados microscópicamente presentaron grados II y III de lesión macroscópica se puede destacar de los procesos inflamatorios: el $72 \%$ se presentaron en forma leve, el 58\% no presentaron infiltrado mononuclear y el $78 \%$ de los tejidos mostraron una dermatitis superficial. Lo anterior puede indicar presentaciones inflamatorias con tendencias a grados leves, que no tienen una relación muy directa con el análisis inicial macroscópico realizado en la planta de sacrificio.

En el $100 \%$ de las muestras evaluadas no se observaron lesiones compatibles con etiología micótica sistémica ni lesiones vasculares con émbolos infecciosos, por lo tanto todos estos resultados sugieren que las lesiones podales estudiadas son esencialmente localizadas y con pocas repercusiones de tipo sistémico.

El trabajo de Gómez, et al (27), mediante estudios físico químicos y microbiológicos informan que muchos de los órganos con estas afecciones son aptos para el consumo humano y no deberían generar pérdida por la retención del producto en las plantas de sacrificio, allí se recomendó evaluar la integridad de la totalidad de los tejidos de los miembros plantares. En respuesta, este estudio obtiene evidencias morfológicas para el 78\% de los casos evaluados que pueden apoyar los análisis del estudio anterior.

De la misma forma, solo en el 22\% de las lesiones evaluadas el aspecto macroscópico y las lesiones microscópicas de las piezas podrían apoyar la decisión de retención por parte de los inspectores, lo cual sugiere discutir, con las entidades de control y vigilancia del Estado, pautas más claras que definan las causas técnicas para la retención de piezas a nivel de planta de sacrificio. 


\section{Agradecimientos}

Esta investigación fue financiada por la Vicerrectoría General de Universidad Abierta y a Distancia de la Universidad Santo Tomás y aprobada en la Convocatoria Interna de Proyectos No 06-2011.

\section{Agradecemos a Pollo Olympico por haber facilitado la infraestructura necesaria para realizar el estudio.}

\section{Referencias}

1. Shepherd EM, Fairchild BD. Footpad dermatitis in poultry. Poult Sci.2010; 89(10):2043-51.

2. Bilgili SF, Hess JB, Donald J, Fancher B. Practical Considerations for Reducing the Risk of Pododermatitis. Aviagen. 2010: $1-7$.

3. Daoust PY. Wadowska D. Kibenge F. Campagnoli RP. Latime K.S. Branson W. R. (2000). Proliferative Pododermatitis Associated with Virus-like Particles in a Northern Gannet. Journal of Wildlife Diseases. 2000; 36(2):378-382.

4. Ask B. Genetic variation of contact dermatitis in broilers May. Poult. Sci. 2010; 89(5):866-875.

5. Stoute ST, Bickford A.A, Walker RL. Mycotic pododermatitis and mycotic neumonía in comermmercial turkey poults in Northern California. Journal of Vaterinary Diagnostic Investigation. 2009; 21(4): 554-557.

6. Nagaraj M, Wilson CA, Hess JB, Bilgili SF. Effect of HighProtein and All-Vegetable Diets on the Incidence and Severity of Pododermatitis in Broiler Chickens. The Journal of Applied Poultry Researchjapr.fass.org.2006;16(3): 304-312.

7. Van der AA. Clay minerals to fight footpad lesions. World Poultry.2008; 24: 15-17.

8. Muniz EC, (2006). Influência da densidade populacional sobre o peso médio, percentual de calo de patas e histomorfometria da bolsa cloacal em aves (Gallus gallus). Dissertaçáo (Mestrado em Ciência Animal), Universidade Federal do Mato Grosso do Sul. Campo Grande, 34.

9. Santos RL, Nunes VA, Bariao NC. Pododermatite de contato em frangos de corte. Arq. Bras. Med. Vet. Zootec. 2002; 54(6):655-658

10. Khodaei M, Yahyai M, Rezaei M, Eidi G, Moazami-godarzi S, Hajkhodadadi I. Determination carcass condemnation causes of broiler chickens (Gallus domesticus) at industrial slaughter house of Shazand, Markazi Province of Iran. Scientific Journal of Animal Science. 2014; 3(5): 91-102.

11. De Jong I. Gunnink H. Van Harn J. Wet litter not only induces footpad dermatitis but also reduces overall welfare, technical performance, and carcass yield in broiler chickens- The Journal of applied poultry research. 2014; 23(4): 51-58.
12. Xavier DB, Broom DM, McManus CM, Torres C, Bernal FE. Number of flocks on the same litter and carcase condemnations due to cellulitis, arthritis and contact foot-pad dermatitis in broilers. British Poultry Science.2010; 51(5):586-591.

13. Borges VP. Principais lesões macro e microscópicas em frangos de corte condenados por caquexia em abatedouro: contribuiçáo ao diagnóstico. Dissertação (Mestrado em Medicina Veterinária), Faculdade de Ciências Agrárias e Veterinárias, Universidade Estadual Paulista. Jaboticabal. 2006:62- 125.

14. Pizarro R, Iicochea D, Reyna S. Efecto del tratamiento de la cama con un aluminosilicato en pollos de carne. Rev. investig. vet. Perú. 2009; 20(2): 213-220.

15. Kyvsgaard N, Bang Jensen H, Ambrosen T. Toft N. Temporal changes and risk factors for foot-pad dermatitis in Danish broilers. Poultry Science. 2014; 92(1): 26-32.

16. Gabanakgosi K, Moreki J C, Nsoso SJ. Tsopito CM. Influence of stocking density on growth performance of family chicks reared up to 18 weeks of age in under an intensive system. Int.J.Curr.Microbiol.App.Sci. 2014; 3(3): 291-302.

17. Remple JD, Adnan A, AL-Ashbal. Raptor Bumblefoot: Another Look at Histopathology and Pathogenesis. In : Raptor Biomedicine .1993.

18. Halliwell WH. 1993. Pododermatitis septica. Disponible en http://www.canariculturacolor.com/foros/showthread. php?t=16175.

19. Hashimoto S, Yamazak k, Obi T. Takase k. Relationship between Severity of Footpad Dermatitis and Carcass Performance in Broiler Chickens. Journal of Veterinary medical Science. 2013; 75(11): 1547-1549.

20. Ekstrand C, Algers B, Svedberg J. Rearing conditions and foot-pad dermatitis in Swedish broiler chickens. Preventive Veterinary Medicine. 1997;31(4):167-174.

21. Pagazaurtundua A, Warriss PD. Measurements of footpad dermatitis in broiler chickens at processing plants. Vet Rec.2005; 158(20):679-82.

22. De Queiroz TV. Anatomopatología e bacteriología da pododermatite em frangos de corte sobinspeção sanitária. dissertação apresentada ao programa de pós-graduação em medicina veterinária da universidade federal fluminense. Niterói Infect. 2009; 137(8):1086-98.

23. Pérez, J. Carrasco, L. Diagnóstico histopatológico de micosis en patología veterinaria. Rev Iberoam Micol. 2000; 17: 18-22.

24. Oviedo, E. (2012). FENAVI, Cali, Colombia, Octubre 2012.

25. Mejía B. En http://patologiaaviarmidiagnostico. blogspot. com/2011_12_01_archive.html.

26. Olias PA, Hauck R, Windhaus H, Van der Grinten E, et al. Articular Aspergillosis of Hip Joints in Turkeys. Avian Diseases.2010; 54:1098-1101.

27. Gómez J. Córdoba G. Guarín, C. Guarín, M. Incidencia de hígado graso y pododermatitis plantar en pollos comerciales en la planta de sacrificio de Pollo Olympico S. A. Rev Ciencia animal. 2011; 4: 41-57. 